\title{
MicroRNA array analysis of the regulation of microRNAs in rats exhibiting hyperplasia of mammary glands
}

\author{
LIYAN GU ${ }^{1}$, QINGXIN XU ${ }^{2}$, HAITAO LIU ${ }^{3}$, JINGRU XIE $^{4}$ and LIDE ZHANG ${ }^{1}$ \\ ${ }^{1}$ Key Laboratory of Ministry of Education for TCM Viscera-State Theory and Applications, \\ Liaoning University of Traditional Chinese Medicine; ${ }^{2}$ Graduate School of Liaoning University of Traditional \\ Chinese Medicine, Shenyang, Liaoning 110847; ${ }^{3}$ The General Hospital of Chinese Armed Police Force, Beijing 100089; \\ ${ }^{4}$ Second Affiliated Hospital of Liaoning University of Traditional Chinese Medicine, Shenyang, Liaoning 110847, P.R. China
}

Received January 19, 2017; Accepted November 24, 2017

DOI: $10.3892 /$ br.2018.1106

\begin{abstract}
Hyperplasia of mammary glands (HMG) is also termed mammary dysplasia. In China, the number of patients suffering from breast hyperplasia is increasing annually. MicroRNAs (miRNAs; length, 19-24 nucleotides), a group of small endogenous non-coding RNAs, post-transcriptionally regulate gene expression via RNA interference and gene silencing pathways. The cause of disease of HMG because remains unclear. Thus, the aim of the present study was to establish comprehensive profile of drug treatments following at different time intervals on rat models of differentially expressed miRNAs, using miRNA microarray data. After scanning the chip, 13 up-regulated and 20 down-regulated miRNAs were identified. MiR-31 and miR-30 exhibited different expression levels between rats exhibiting mammary gland hyperplasia treated with or without Jiedu Capsule water solution once a day for 4 weeks, and the two demonstrated a strong association with HMG and breast cancer. Therefore, the functions of these miRNAs may provide the basis for further investigation of HMG.
\end{abstract}

\section{Introduction}

Hyperplasia of mammary gland (HMG) is also termed mammary dysplasia. It does not involve inflammation or tumor proliferative lesions. It is a disease characterized by basic pathological changes in breast acinar and ductal epithelial cells, as well as fibrous connective tissue hyperplasia. The peak age of breast hyperplasia is 35-45 years (1). Mammary gland hyperplasia is a common disease in women of child-bearing period,

Correspondence to: Dr Lide Zhang, Key Laboratory of Ministry of Education for TCM Viscera-State Theory and Applications, Liaoning University of Traditional Chinese Medicine, 79 Chong shan Road, Shenyang, Liaoning 110847, P.R. China

E-mail: zhldtcm@163.com

Key words: hyperplasia of mammary glands, miRNA array, profiling, molecular biological mechanism accounting for $75 \%$ of all breast diseases, is considered to be precancerous lesions of the breast. In China, HMG is increasing annually and regarded as a precancerous lesion, which causes widespread concern. According to modern medicine, breast tissue is the subject organ of a variety of hormone actions. The physiological function of the breast tissue is controlled by the hypothalamic pituitary ovary axis. Increased sensitivity of any hormone, or breast tissue to hormones, is the primary cause of breast hyperplasia (2).

MicroRNA (miRNA) is a class of non-encoding RNA (length, $22 \mathrm{nt}$ ), which regulates gene expression at the post-transcriptional level (3). After the stem loop structure of precursor miRNA is spliced by two RNase III enzymes, Drosha and Dicer, the mature functional miRNA is formed (4). The mature miRNAs are incorporated into protein-coding transcripts and represses the transcript translation or degrades the mRNA in mammals (5). Consequently, this regulates various biological processes, including development, reproduction, apoptosis, proliferation, pathogenesis, and lipometabolism $(6,7)$. Thus, the abnormal expression of miRNAs may cause various diseases, such as cardiac disease and cancer (8). A strong association between miRNA and breast cancer has been reported in the literature. However, in reviewing the literature, little data was identified regarding the association between HMG and miRNAs. The aim of the present study was to use a microRNA array to analyze the role of microRNAs in rats exhibiting hyperplasia of the mammary glands.

\section{Materials and methods}

Sample preparation. A total of six Sprague Dawley specific pathogen-free female rats (weight, $240 \mathrm{~g}$; age, 12 weeks) that were not pregnant were used in the present study. The rats were obtained from Beijing HFK Bio-science Co. Ltd. (Beijing, China) and reared in the animal center of Liaoning University of Traditional Chinese Medicine (Shenyang, China). The rats were housed at $21-25^{\circ} \mathrm{C}$ and $35-50 \%$ humidity under a $12-\mathrm{h}$ light/dark cycle and were provided feed and sterilized water in a 1:2 ratio twice a day. All animals were fed with a standard laboratory diet and tap water was provided ad libitum. The animals were placed in the experimental room $24 \mathrm{~h}$ before behavioral testing for acclimatization. The rats were housed 
for five days to allow adaptation to the laboratory conditions. On the sixth day, intramuscular $0.5 \mathrm{mg} / \mathrm{kg} / \mathrm{d}$ estradiol benzoate (Wuhan Amyjet Scientific, Inc., Wuhan, China) for 20 days, and $5 \mathrm{mg} / \mathrm{kg} / \mathrm{d}$ progesterone (An Apoptosis and Epigenetics Company, A8509) was injected at day 26 for 5 days. The model cycle was 25 days, which resulted in induction of the mammary gland hyperplasia model. After modeling successfully, the rats were divided into two groups. A control (MA) group was treated with physiological saline $(2 \mathrm{ml})$ once a day for 4 weeks, while a treatment (GA) group underwent 4 weeks of daily treatment with 0.15625g Jiedu Capsule (Radix Bupleuri $15 \mathrm{~g}$, Astragalus $15 \mathrm{~g}$, honeysuckle $15 \mathrm{~g}$, forsythia $15 \mathrm{~g}$, cantharidin $15 \mathrm{~g} ; 0.5 \mathrm{~g}$ powder/capsule) per $1 \mathrm{~kg}$ body weight dissolved in $2 \mathrm{ml}$ distilled water by intragastric administration. Following the treatment period, experimental samples were obtained by surgery. The animals were anesthetized by intraperitoneal injection with $10 \%$ chloral hydrate aqueous solution according to a $0.3 \mathrm{ml} / 100 \mathrm{~g}$ dose. The mammary glands of rats were numbered according to order from head to tail. The whole third pair of papillae was excised and placed in an Eppendorf tube and stored at $-80^{\circ} \mathrm{C}$ for RNA isolation. The study protocol was approved by Animal Ethical Inspection Review Board of Liaoning University of Traditional Chinese Medicine, and was conducted according to their ethical principles of animal welfare.

RNA isolation and preparation. Total RNA was extracted from the mammary papilla samples using an mirVana ${ }^{\mathrm{TM}}$ miRNA Isolation kit (Thermo Fisher Scientific Inc., Waltham, MA, USA) according to the manufacturer's instructions. The total RNA quantity and purity were analyzed using a Bioanalyzer 2100 and RNA 6000 Nano LabChip kit (Agilent Technologies, Inc., Santa Clara, CA, USA) with RNA integrity number $\geq 5.0$ and an SSP-3000 Nanodrop spectrophotometer (Infinigen Biotech, Inc., Hacienda Heights, CA, USA) at a wavelength of $260 \mathrm{~nm}$.

Microarray hybridization and data analysis. The Mouse \& Rat miRNA OneArrayV6 (Phalanx Biotech Group, Hsinchu, Taiwan) contains a total of 4,104 probes, including 144 experimental control probes, 1,907 unique mouse miRNA probes and 728 rat miRNA probes. Each probe on the microarray chip has a chemically modified segment encoding nucleotides complementary to the target based on miRBase version 17 (http://www.mirbase.org/). Rat genome-wide miRNA microarray analysis was performed by Beijing Ori-Gene Science and Technology Corp., Ltd. (Beijing, China). Briefly, fluorescent targets were prepared from $2.5 \mu \mathrm{g}$ total RNA using the miRNA ULS Labeling kit (Kreatech Diagnostics, Amsterdam, The Netherlands). Labeled miRNA targets were enriched using NanoSep 100K (Pall Corporation, Port Washington, NY, USA), enriched products were hybridized to the Mouse \& Rat miRNA One Array v3 in Phalanx hybridization buffer using the One Array Hybridization Chamber, and hybridized overnight at $37^{\circ} \mathrm{C}$, non-specifically bound targets were removed by three washing steps (first wash, at $37^{\circ} \mathrm{C}$ for $5 \mathrm{~min}$; second wash, at $37^{\circ} \mathrm{C}$ for $5 \mathrm{~min}$ and at $25^{\circ} \mathrm{C}$ for $5 \mathrm{~min}$; and third wash included 20 rinses at $37^{\circ} \mathrm{C}$ ). Then, the slides were dried and scanned using an Axon 4000B scanner (Molecular Devices, LLC, Sunnyvale, CA, USA).

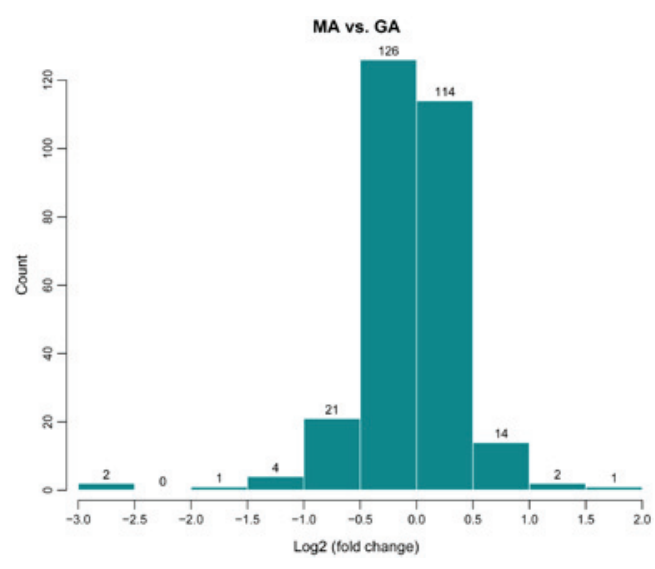

Figure 1. Differences in gene expression between the GA and MA groups. The MA group was treated with physiological saline $(2 \mathrm{ml})$ once a day for 4 weeks, while the GA group underwent 4 weeks of daily treatment with 9.375 g Jiedu Capsule.

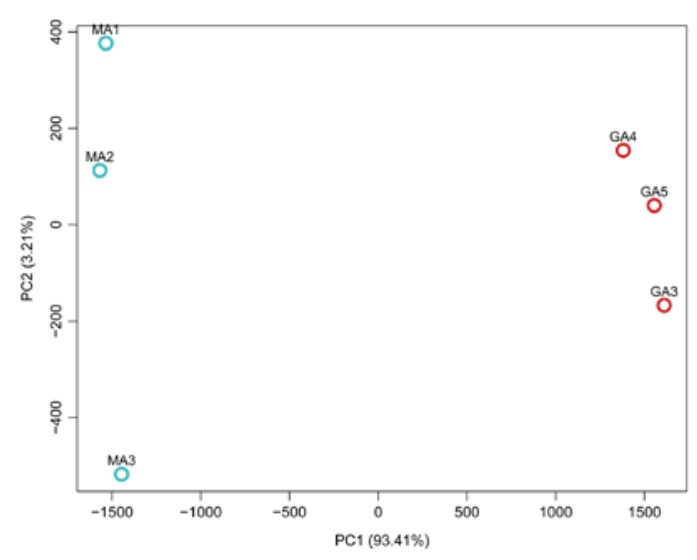

Figure 2. Principal component analysis. This two-dimensional display includes horizontal coordinate, $\mathrm{PC} 1$, which indicate the first principal component, and the vertical coordinate, $\mathrm{PC} 2$, which indicate the second principal component. The gene expression of MA and GA in the different treatment groups was distinguished in the dimension of PC1. MA1-3, control samples; GA3-5, treatment samples.

Multiple sample analyses were conducted, including normalization, data adjustment, analysis of variance and clustering. The fluorescence signal intensity of Cy5 of each point was analyzed by GenePix 4.1 software (Molecular Devices, LLC) and processed using the $\mathrm{R}$ program. The data were initially analyzed by subtracting the background, and then spots for which the flag was $<0$ were filtered out, and spots that passed these criteria were normalized using a $75 \%$ media scaling normalization method. Normalized spot intensities were converted into gene expression log2 ratios for the MA and GA groups. Spots with $\log 2$ ratios $\leq-1$ or $\geq 1$ and $\mathrm{P}<0.05$ were selected for further analysis. The differential expression was analyzed using average linkage algorithm to cluster and Pearson correlation as a measure of similarity.

\section{Results}

Upregulated miRNA targets in microarray analysis. In order to investigate the difference of gene expression between the 


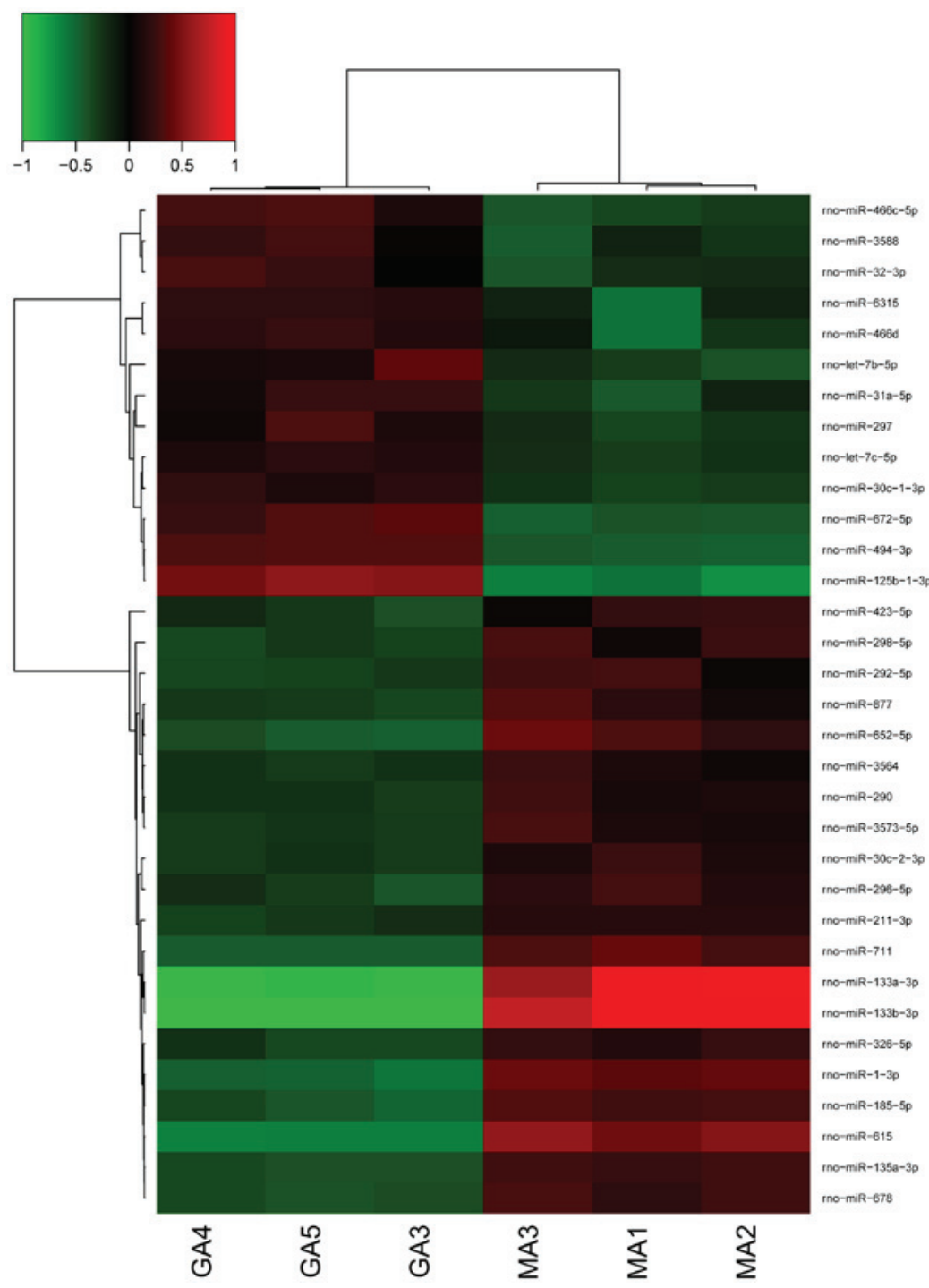

Figure 3. Hierarchical cluster analysis of differentially expressed circulating miRNAs of mice in the MA and GA groups ( $\mathrm{n}=3$ per group). All six samples were clustered according to MA or GA group origin, and 3 samples in each of the MA and GA groups were clustered into separate classes. MA1-3, control samples; GA3-5, treatment samples.

GA and MA groups, the differences in the distribution of all detected gene probe signals were statistically analyzed. The screening conditions of differentially expressed genes are $\log 2$ fold-change $\geq 0.585$ and $\mathrm{P}<0.05$ (9). The results of the miRNA microarray (Fig. 1) indicated 13 upregulated miRNAs (rno-let-7b-5p, rno-let-7c-5p, rno-miR-31a-5p, rno-miR-297, rno-miR-466c-5p, rno-miR-30c-1-3p, rno-miR-672-5p, rno-miR-125b-1-3p, rno-miR-32-3p, rno-miR-3588, rno-miR-466d, rno-miR-494-3p and rno-miR-6315) and 20 downregulated miRNAs (rno-miR-30c-2-3p, rno-miR-1-3p, rno-miR-292-5p,rno-miR-133a-3p, rno-miR-877, rno-miR-615, rno-miR-133b-3p, rno-miR-185-5p, rno-miR-135a-3p, rno-miR-678, rno-miR-711, rno-miR-296-5p, rno-miR-298-5p, rno-miR-326-5p,rno-miR-211-3p,rno-miR-290,rno-miR-3564, rno-miR-3573-5p, rno-miR-423-5p and rno-miR-652-5p) in the drug treatment group (GA).

Principal component analysis (PCA). A PCA method (10,11) was used to assess differences between the transcriptomes of rats treated with Jiedu Capsule and controls. PCA1 and PCA2 represented the top two dimensions of the genes exhibiting differential expression among the samples, which accounted for 93.41 and $3.21 \%$ of the differentially expressed genes, respectively (Fig. 2).

Cluster analysis of differentially expressed genes. Cluster analysis is a method of simplifying data by data modeling, the purpose is to obtain the distribution of data and the characteristics of each cluster, and the association between sample clusters is described by the distance index. An in-depth analysis of differentially expressed genes was conducted, which were identified with the criteria of $\mathrm{P}<0.05$ and expression fold change $>2.0$. Unsupervised hierarchical clustering analysis was used to investigate the similarity of the whole gene expression between the experimental samples. Cluster analysis identified that all the six samples were clustered into two groups (Fig. 3); the 3 samples in the control group were clustered into one class while those in the treatment group were clustered into another class. This indicates that the trend of gene expression is consistent. 


\section{Discussion}

Hyperplasia of the breast is a common disease in women aged 20-55 years and is associated with an increased risk of developing cancer. Breast cancer is the second most common type of cancer in women, and is an important cause of mortality in most frequent cancer of cancer-related of death worldwide and generates a large economic burden (12). A previous study identified that women with atypical hyperplasia $(\mathrm{AH})$ had a four- to five-fold increase in breast cancer risk (13). Furthermore, a strong association between $\mathrm{AH}$ and breast cancer was identified (14).

In the present study, miRNA microarray scanning was performed on the breast tissue of rats that had undergone drug treatment. It was identified that the expression levels of the 13 miRNAs in the GA and MA groups were markedly different; the expression levels of 13 miRNAs in group GA was greater than in group MA, and the expression levels of 20 miRNAs was significantly decreased in the GA group, and the difference was statistically significant.

The experimental results and numerous previous studies reported that miR-31 is over expressed in metastatic breast cancer (15). Rasheed et al (16) and Valastyan et al (17) identified that the primary function of miR-31 promotes multiple oncogenic phenotypes, including proliferation, motility and invasion of cancer cells. Another notable finding was that manipulation of miR-31 expression levels may be used to modulate senescence-associated pathological conditions, such as cancer and the ageing process (18). The current study demonstrated that the expression level of miR-31 in the GA group was significantly greater than in the MA group.

By contrast, the expression level of miR-30 in the GA group was lower than that in the MA group. The current study indicated that breast cancer cells grown under non-attachment conditions display a unique pattern of miRNA expression, compared with the parental cells, as highlighted by a marked low expression of miR-30 family members. Ouzounova et al (19) showed that miR-30a regulates non-attachment growth. Furthermore, it was demonstrated that miR-30c may regulate the GATA binding protein 3 gene in breast cancer at the transcriptional level (20).

Thus, certain genes found in the present study are in agreement with the existing studies. However, further studies are required to verify other miRNAs.

In conclusion, the present study identified the expression profile of circulating miRNAs in the model group and GA group. Following a scan of the chip, 13 upregulated and 20 downregulated miRNAs were identified, and the functions of these miRNAs were described. It was demonstrated that the expression levels of miRNAs are closely associated with biological processes, such as mammary gland development and cell proliferation. The present study provides a basis upon which to further investigate the molecular mechanism of HMG. However, there was no validation of the differentially expressed miRNAs, and future work is required to confirm their function. The results of the present study also suggested that hyperplasia of mammary glands occurs as a result of complex and dynamic processes regulated by numerous factors, including multiple miRNAs. Therefore, the functions and significance of the differentially expressed miRNAs identified in this study require further investigation. Future work should aim to functionally analyze miRNAs associated with hyperplasia of mammary glands and their corresponding target genes or gene regulatory networks, in an expanded sample size to further verify results.

\section{Acknowledgements}

The current study was partially supported by the Science Public Welfare Research Foundation of Liaoning Province, China (2014001021), the Key Laboratory of Ministry of Education for TCM Viscera-State Theory and Applications Foundation (zyzx 1609), the Natural Science Foundation of Liaoning Province, China (20170540612), the Educational Commission of Liaoning Province, China (L201717), the Science and Technology Planning Project of Shenyang, Liaoning, China (18-013-0-11) and the College Students' innovation and entrepreneurship training program project, Liaoning University of Traditional Chinese Medicine, (201610162034 and 201710162000088).

\section{References}

1. Chang XJ, Zhou J, Zhang S, Chen J, Chen CM, Wang ZZ and Xiao W: Effects of guizhi fuling capsule on sex hormone levels and breast issue morphology of mammary gland hyperplasia model rats. Zhongguo Zhong Yao Za Zhi 39: 4139-4142, 2014 (In Chinese).

2. Liu B and Guan ZH: Radix bupleuri Extract Inhibits Hyperplasia of Mammary Gland in Rats. Trop J Pharm Res 15: 293, 2016.

3. Ekmekci C, Mousses S and Tuzmen S: MicroRNA (miRNA), involvement in aberrant promoter methylation facilitating tumor progression. Cancer Res 67: 1046-1046, 2007.

4. Rahimi G, Jafari N, Khodabakhsh M, Shirzad Z and Dogaheh HP: Upregulation of microRNA processing enzymes Drosha and Dicer in gestational diabetes mellitus. Gynecol Endocrinol 31: 156-159, 2015.

5. Mallick B and Ghosh Z: Probing Evolutionary Biography of MicroRNAs and Associated Factors. Curr Genomics 13: 144-152, 2012.

6. Bae S, Lee EM, Cha HJ, Kim K, Yoon Y, Lee H, Kim J, Kim YJ, Lee HG, Jeung HK, et al: Resveratrol alters microRNA expression profiles in A549 human non-small cell lung cancer cells. Mol Cells 32: 243-249, 2011.

7. Ambros V: The functions of animal microRNAs. Nature 431: 350-355, 2004.

8. Martins IJ: Induction of NAFLD with Increased Risk of Obesity and Chronic Diseases in Developed Countries. Open J Endocr Metab Dis 04: 90-110, 2014.

9. Badri H, Monsieurs P, Coninx I, Nauts R, Wattiez R and Leys N: Temporal Gene Expression of the Cyanobacterium Arthrospira in Response to Gamma Rays. PLoS One 10: e0135565, 2015.

10. Vyas S and Kumaranayake L: Constructing socio-economic status indices: How to use principal components analysis. Health Policy Plan 21: 459-468, 2006.

11. Lee C, Abdool A and Huang CH: PCA-based population structure inference with generic clustering algorithms. BMC Bioinformatics 10 (Suppl 1): S73, 2009.

12. Venkatadri R, Muni T, Iyer AKV, Yakisich JS and Azad N: Role of apoptosis-related miRNAs in resveratrol-induced breast cancer cell death. Cell Death Dis 7: e2104, 2016.

13. Dupont WD and Page DL: Risk factors for breast cancer in women with proliferative breast disease. N Engl J Med 312: 146-151, 1985.

14. Dupont WD, Parl FF, Hartmann WH, Brinton LA, Winfield AC, Worrell JA, Schuyler PA and Plummer WD: Breast cancer risk associated with proliferative breast disease and atypical hyperplasia. Cancer 71: 1258-1265, 1993.

15. Cho JH, Dimri M and Dimri GP: MicroRNA-31 is a transcriptional target of histone deacetylase inhibitors and a regulator of cellular senescence. J Biol Chem 290: 10555-10567, 2015. 
16. Rasheed SA, Teo CR, Beillard EJ, Voorhoeve PM, Zhou W, Ghosh S and Casey PJ: MicroRNA-31 controls G protein alpha-13 (GNA13) expression and cell invasion in breast cancer cells. Mol Cancer 14: 67, 2015.

17. Valastyan S, Reinhardt F, Benaich N, Calogrias D, Szász AM, Wang ZC, Brock JE, Richardson AL and Weinberg RA: A pleiotropically acting microRNA, miR-31, inhibits breast cancer metastasis. Cell 137: 1032-1046, 2009.

18. Cho JH, Dimri M and Dimri GP: MicroRNA-31 is a transcriptional target of histone deacetylase inhibitors and a regulator of cellular senescence. J Biol Chem 290: 10555-10567, 2015.

19. Ouzounova M, Vuong T, Ancey PB, Ferrand M, Durand G, Le-Calvez Kelm F, Croce C, Matar C, Herceg Z and Hernandez-Vargas H: MicroRNA miR-30 family regulates non-attachment growth of breast cancer cells. BMC Genomics 14: $139,2013$.
20. Bockhorn J, Dalton R, Nwachukwu C, Huang S, Prat A, Yee K, Chang YF, Huo D, Wen Y, Swanson KE, et al: MicroRNA-30c inhibits human breast tumour chemotherapy resistance by regulating TWF1 and IL-11. Nat Commun 4: 1393, 2013.

This work is licensed under a Creative Commons Attribution-NonCommercial-NoDerivatives 4.0 International (CC BY-NC-ND 4.0) License. 